\title{
CIVIL AVIATION PILOT EDUCATION, COACHING AND BEYOND: BUILDING CAPACITY IN THE COCKPIT
}

\author{
Olja Čokorilo ${ }^{1}$ \\ ${ }^{1}$ University of Belgrade, Faculty of Transport and Traffic Engineering, Vojvode Stepe 305, 11000 \\ Belgrade, Serbia
}

Received 2 August 2019; accepted 13 December 2019

\begin{abstract}
Development capacities and improvement of pilot education in the future will require the application of modern methods and models. These models will guide the development process and the future career of professional pilots within the framework of safety culture and Safety Management System (SMS). The development process aims to enable an adequate balance of the current performance of the individual and the strengthening of his skills and abilities in relation to the principles of just culture. Similar to the Reason model, the entire process can be directed through four pillars: inputs, people, process and outputs. Modern coaching principles allow selection of appropriate models based on individuals rather than on the process. To this end, modern coaching models GROW and Co-Active will be considered in this paper. The paper aims to highlight the importance of introducing coaching in the education of professional civil aviation pilots and the expected positive effects on the development of a professional and safety culture in aviation industry.
\end{abstract}

Keywords: pilot, education, safety culture, coaching.

\section{Introduction}

By enhancing the capacity building efforts of each individual pilot, it is possible to improve the common synergy of the entire aviation professional staff and even of the global aviation industry in terms of improving safety. Observed over a longer period, development and implementation of coaching techniques into the pilot's educational process can lead to a reduction in human error in aviation operations. This chapter shows how a coaching process can influence the attitudes, values, beliefs, behavior and daily work of pilots based on appropriate techniques. Also, it considers the importance of selecting the appropriate effective coaching model tailored to the individual according to his performances.
Adopting new knowledge is one of the key elements in educating professional pilots. During the learning process, professional and personal development are two main categories. Common in both cases are new knowledge-oriented processes management, work with certain categories of people and aviation staff, risk management, safety and just culture, etc. The most known models that could be used in analysis from the aviation industry are: SHELL (software, hardware, environment, liveware and other liveware) and Reason model.

In the process of couching professional pilots, in addition to new knowledge and understandings, the choice of a personal coaching model should certainly include previous life and professional experiences based on: specific experience in

${ }^{1}$ Corresponding author: o.cokorilo@sf.bg.ac.rs 
the field of safety culture and the principles of safety management; the possibility of analyzing the causes of occurrence of certain events / occurrences from the perspective of national, organizational, professional and safety culture, etc.

\section{Human Factor Implications on Safety Risk Management}

People working in aviation are crucial for safe and cost-effective air traffic management which is a key enabler to the benefits of aviation. Human performance and limitations based on ability and skills of flight and cabin crew members, ATM controllers and maintenance and repair personnel help in the prevention of numerous accidents and incidents on a daily basis. However, despite keeping a record of and monitoring global safety issues, many studies define a human factor as the cause of at least $2 / 3$ accidents in commercial aviation (Čokorilo, 2013; Čokorilo and Dell'Acqua, 2013). Leading theoreticians and researchers in the field of human factors believe that almost $70 \%$ of all aviation accidents are caused by human error (Shappell and Wiegmann, 2009).

Table 1 shows the distribution of the main causes of accidents by decades, where the human error has the predominant role.

Table 1

Causes of Fatal Accidents per Decade (\%)

\begin{tabular}{|l|c|c|c|c|c|c|c|}
\hline Cause & $\mathbf{1 9 5 0}$ & $\mathbf{1 9 6 0}$ & $\mathbf{1 9 7 0}$ & $\mathbf{1 9 8 0}$ & $\mathbf{1 9 9 0}$ & $\mathbf{2 0 0 0}$ & Average \\
\hline Pilot Error & 40 & 32 & 24 & 25 & 27 & 26 & 29 \\
\hline Pilot Error (Weather Conditions) & 11 & 18 & 14 & 17 & 21 & 17 & 16 \\
\hline Pilot Error (Mechanical Failure) & 7 & 5 & 4 & 2 & 4 & 3 & 4 \\
\hline Total Pilot Errors & 58 & 55 & 42 & 44 & 52 & 46 & 50 \\
\hline $\begin{array}{l}\text { Other Human Errors } \\
\begin{array}{l}\text { ATM Controllers, Inadequate Aircraft } \\
\text { Loading, Inadequate Maintenance, etc.) }\end{array}\end{array}$ & 0 & 8 & 9 & 6 & 8 & 8 & 7 \\
\hline Weather Conditions & 16 & 10 & 13 & 15 & 9 & 9 & 12 \\
\hline Mechanical Failure & 21 & 20 & 23 & 21 & 21 & 28 & 22 \\
\hline $\begin{array}{l}\text { Sabotage (Explosive, Unlawful } \\
\text { Interference, Terrorist Attacks, etc.) }\end{array}$ & 5 & 5 & 11 & 13 & 10 & 9 & 9 \\
\hline Other Causes & 0 & 2 & 2 & 1 & 0 & 1 & 1 \\
\hline
\end{tabular}

Although the focus is often on the pilot in command, they are not the only threat. However, pilots are on the last defense line and are often in a position to identify and correct errors that can cause aircraft accidents and incidents. On the other hand, statistics show that pilots generate the most errors that often lead to aviation accidents.

Studies show that human errors that result in aircraft accidents can be divided into several categories: pilot errors (33\%), copilot errors (17\%), ATM controller errors (17\%), system design errors (9\%), maintenance errors (8\%), crew errors (6\%) and other errors (10\%).

In contemporary research, safety attention is largely focused on understanding the decision-making process, especially to how people react in different operational situations and what is their interaction with new technologies and improvements 
in aviation safety systems. The way that "manages" people affects their behavior, which further affects their performance of critical tasks. Investigations of black boxes (after aircraft accident) clearly indicate that external disturbances must be minimized and strictly performed in accordance with the prescribed procedures during the flight, especially during critical flight phases (taxiing, take-off, approach and landing).

Basically, the problem is that people often make the wrong decisions. Basically, there are three reasons for making inappropriate or wrong decisions: they have incomplete information, they use inaccurate or irrelevant information, or poorly process the information they have. Miller's law (Miller, 1956) explains the fact that people have limited capability of processing the information (the number of objects an average person can store in a working memory is $7 \pm 2$ ). This number increases when the pilot uses both visual and audio channels, since the likelihood of an aircraft accident occurring increases: during a high workload, a working saturation period, or when there is overload of one or more information processing channels at the observed pilot (Rodrigues and Cusick, 2012).

In order to reduce the scope of work in critical workload situations, new pilots learn the strategy of the current "neglect" of less important tasks, in order to focus on the most important task during the flight procedure. (FAA, 2009) provides the general rule as: aviate, navigate, and communicate. In other words, during the emergency situation, first, keep the airplane in the air, i.e. pilot, then if the circumstances allow you to operate an airplane, i.e. keep it at the specified flight course, height and speed; and finally communicate with the ATC.
Understanding the human factor is very important in systems where people regularly interact with sophisticated machines and technologies and in industries where accidents caused by human error can have catastrophic consequences. Technical decisions related to the design, manufacture, regulations and operations of aircraft are based on complex scientific principles covered by multi-discipline approach. Human abilities cannot be accurately measured and human factor research requires much more time and attention than most other aviation research.

The consideration of human factors has particular importance in Safety Risk Management as people can be both a source and a solution of safety risks by (ICAO, 2018):

- Contributing to an accident or incident through variable performance due to human limitations;

- Anticipating and taking appropriate actions to avoid a hazardous situation;

- Solving problems, making decisions and taking actions to mitigate risks.

It is therefore important to provide multi disciplinary approach and to involve experts with appropriate human factors expertise in the identification, assessment and mitigation of safety risks. Safety Risk Management principles are based on adequate hazard understanding, identification, analysis, documentation and addressing safety risks. Appropriate generic hazard is the most important for establishing risk management processes. Safety Risk Management requires all aspects of safety risks to be addressed, including those related to humans. Assessing the risks associated with human performance is more complex than risk factors associated with technology and environment since: 
- Human performance is highly variable, with a wide range of interacting internal and external influences to the individual. Many of the effects of the interaction between these influences are difficult, or impossible to predict;

- The consequences of variable human performance will differ according to the task being performed and the context.

Therefore, modeling human performance and limitations is valuable in the identification and assessment of safety risks. Therefore, human factors expertise is a key driver in the identification and assessment of safety risks whereas determination of safety risks severity and probability could be simplified.

\section{Modeling Human Performance and Limitations}

Contemporary models for modeling human performance and limitations, which are used to prevent aircraft accidents are presented below.

\subsection{SHELL Model}

In order to understand the human contribution on aviation safety and to support the human performance due to achieving the basic goals of the system, it is necessary to understand how different components and characteristics in the operating context can be influenced by human performance, as well as the interaction between the components, characteristics and specificity of the system, and people.

A simple but visually powerful, conceptual tool for analyzing the components and characteristics of the operational context and their possible interactions with people is the SHELL model. This model can be used to visualize the relationships between different components and characteristics of the aviation operational context. SHELL model illustrates the relationship between human (at the centre of the model) and workplace components (ICAO, 2018). The name of the SHELL model originates from the initial letters of its four components:

- Software-S (procedures, training, support, etc.);

- Hardware-H (machines and equipment);

- Environment-E (operational circumstances in which the rest of the L-H-S system operate);

- Liveware-L (other humans in the workplaces).

Figure 1 describes the SHELL model. This block diagram is intended to provide a basic understanding of the individual's relation to the components and characteristics of the workplace. There are people in the SHELL model center. Although people are very flexible, there are significant variations in their work. People are not standardized to the same extent as hardware, so the edges of that block are not simple and straightforward. People do not have a perfect connection with the different components in the working environment. In order to avoid tensions that can compromise human performances, the effects of border irregularities between different SHEL blocks and the central "Liveware" block must be clearly understand. A number of different factors are responsible for uneven edges on the "Liveware" block. Some of the most important factors affecting the human performance are: physical, physiological, psychological and psychosocial factors (ICAO, 2005): 
- Physical factors include the individual's physical capabilities to perform the required tasks (e.g. strength, height, reach, vision and hearing);

- Physiological factors include those factors which affect the human's internal physical processes, which can compromise the crew's physical and cognitive performance (e.g. oxygen availability, general health and fitness, disease or illness, tobacco, drug or alcohol use, personal stress, fatigue or pregnancy);

- Psychological factors include those factors affecting the psychological preparedness of the individual to meet all the circumstances that might occur during a flight (e.g. adequacy of training, knowledge and experience, visual illusions and workload). The individual's psychological fitness for duty includes motivation and judgment, attitude towards risky behavior, confidence, stress, etc.;

- Psycho-social factors include all those external factors in the individual's social system that bring pressure to bear on them, both in their work and their nonwork environments (e.g. argument with a supervisor, labor-management disputes, a death in the family, personal financial problems or other domestic tension).

\subsection{Generic Error-Modeling System (GEMS)}

The Generic Error-Modeling System (GEMS) provides the framework for the integration of different unintentional error categories (slips, lapses and mistakes) and the three levels of performance (skill, rule, knowledge). This two-dimensional approach provides:
- To gain a deeper understanding the nature of mistakes and to distinguish between rule-based mistakes and knowledge-based mistakes;

- To appreciate the details of the differences among error types;

- To appreciate how errors can be considered the "other side of the coin" of those cognitive processes that allows to act quickly or find creative solutions;

- To anticipate when and in what conditions, a certain type of error may occur.

GEMS treats the errors that can occur at each operational level:

- Skill-based (SB): slips and lapses as errors of inattention or misplaced attention;

- Rule-based (RB): mistakes as a result of picking an inappropriate rule or caused by misconstrued view of state, overzealous pattern matching, frequency gambling, etc.;

- Knowledge-based (KB): mistakes generate due to incomplete or inaccurate understanding of system, confirmation bias, overconfidence, cognitive strain, etc.

Errors can arise as a result of operating at the wrong level (people do not want to move from the rule-based level to the knowledgebased level, even when the rules do not work.

\subsection{Heinrich Pyramid}

Heinrich's Law (Heinrich, 1941) defines relationship between occurrences and more serious incidents and accidents: "In a workplace, for every accident that causes a major injury, there are 29 accidents that cause minor injuries and 300 accidents that 
cause no injuries". This concept is pictorial presented as a pyramid (1:30:300).

Heinrich's law is based on probability and assumes that the number of accidents is inversely proportional to the severity of those accidents. It leads to the conclusion that minimizing the number of minor incidents will lead to a reduction in major accidents, which is not necessarily the case (Heinrich, 1941).

\subsection{Human Factor Analysis and Classification System (HFACS)}

The Human Factors Analysis and Classification System-HFACS is a broad human error framework that was originally used by the US Air Force to investigate and analyze human factors aspects of aviation. HFACS is heavily based upon SwissCheese model (Reason, 1990). The HFACS framework provides a tool to assist in the investigation process and target training and prevention efforts. Investigators are able to systematically identify active and latent failures within an organization that culminated in an accident. The goal of HFACS is not to attribute blame, it is to understand the underlying causal factors that lead to an accident (Wiegmann and Shappell, 2017).

The HFACS framework treats human error at each of four levels of failure:

- Unsafe acts of operators (e.g. flight crew, ATC controllers)-errors and violations;

- Preconditions for unsafe actsenvironmental factors, personnel factors and condition of operators;

- Unsafe supervision-inadequate supervision, plan inappropriate operation, fail to correct known problem, supervisory violation;
- Organizational influences-resource management, organizational process, organizational climate.

When an accident or an incident occurs, proper use of HFACS allows the safety analyst to identify a specific human error that has occurred at different levels of the organization. It can be noted that the investigation of an accident is focused on "what" happened and in particular "why" it happened in the organization. The next step in this complex process is to consider a multi-stage framework of factors that are potential causes of an accident. (Shappell and Wiegmann, 2009) propose 5 intervention strategies for human error control in the following:

- Organization / administration;

- People / crew;

- Technology / engineering;

- Tasks / operations;

- Operational / physical environment.

As measures against unsafe acts have been previously considered, this relationship becomes the Human Factors Intervention Matrix (HFIX). HFACS classification methodology and HFIX intervention matrix are modern human factor analysis tools designed for sophisticated organizations with high quality data and trained staff. They provide a very useful framework for aviation accident investigators who use them to study the organization and its role in causing accidents.

\subsection{Swiss-Cheese Model}

The Swiss-Cheese model of accident causation, originally proposed by James Reason, likens human system defenses to a series of slices of randomly-holed Swiss 
cheese arranged vertically and parallel to each other with gaps in-between each slice (Reason, 2016). The failure of the system as a whole comes when the holes in all parts are currently aligned, allowing the "trajectory of the accident", so that the hazards pass through the holes on all defenses, causing the accident (Figure 1). The model assumes that the most accidents can be traced to one or more of four levels of failure:

- Organizational influences;

- Unsafe supervision;

- Preconditions for unsafe acts;

- The unsafe acts themselves.

According to the Reason model, in order to generate accident, it is necessary to have a common action of several factors that are individually insufficient to violate the defense system. A complex system such as aviation is extremely welldefended from several levels of defense (technology, regulation and training), and therefore individual failures are not of the main importance in the aviation system. Equipment failures or operational errors are never a cause for safety breach, but are only triggers. Violation of safety rules represents the delayed consequences of decisions made at the highest level of management and remain hidden until their effects or harmful potentials are not activated by a specific set of operational circumstances. Under these circumstances, human error or active failures at the operational level act as triggers of latent conditions and lead to violation of the safety system defenses.

Active failures include errors and violations of the rules and procedures and have an immediate adverse effect. Generally, these are unsafe actions. Active failures are generally associated with the personnel who (not)provides actions (pilots, ATC controllers, etc.) which can result by the inappropriate outcome.

Active operational human errors occur in an operational context that includes hidden, latent conditions. Latent conditions are the conditions present in the system quite long before there is a harmful outcome. Basically, these latent conditions are generally not considered as harmful, as they are not seen as errors / failures even at the beginning of the process. Latent conditions become evident when the safety system or defense is violated.

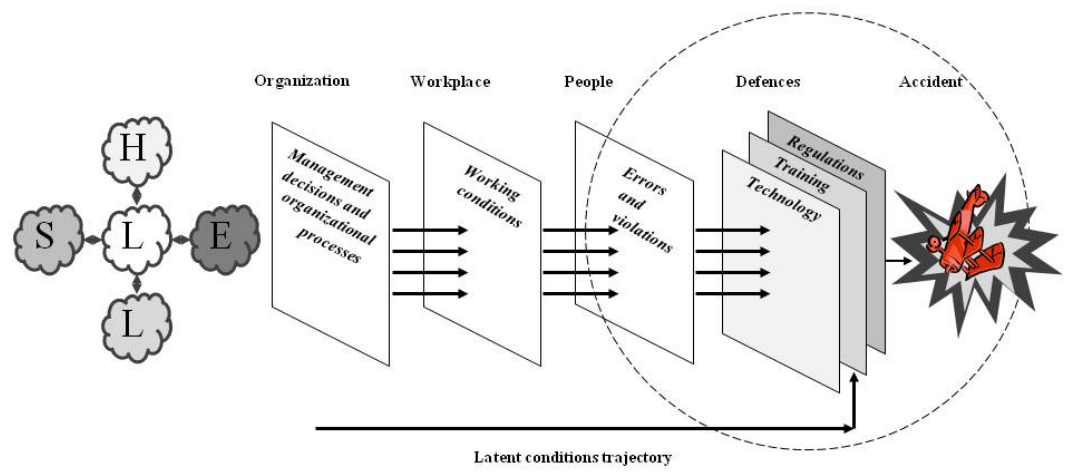

Fig. 1.

SHELL Model vs. Swiss-Cheese Model 
Figure 1 illustrates how the Swiss-Cheese model assists in understanding the interplay of organizational and managerial factors in accident causation. Multiple layers of defense are built into the aviation system to protect against variations in human performance or decisions at all levels of the organization. But each layer typically has weaknesses, depicted by the holes in the slices of "Swiss cheese". Sometimes all of the weaknesses align (represented by the aligned holes) leading to a breach that penetrates all defensive barriers and may result in a catastrophic outcome. The Swiss-Cheese model represents how latent conditions are ever present within the system and can manifest through local trigger factors.

\section{Human Error and Error Management in Aviation}

Human error is cited as being a causal or contributing factor in the majority of aviation occurrences. Although competent personnel commit such errors, accident reports show that they did not plan an accident. Errors are not some type of aberrant behavior, they are a natural bi-product of virtually all human endeavor. Error must be accepted as a normal component of aviation system where humans and technology interact.

The main factors contributing to human errors and to accidents are: organizational factors, equipment design, procedures, personal factors, training, culture and other factors. Aviation companies provide several error management strategies in practice: error prevention, error reduction, error detection, error recovery and error tolerance. Therefore it is of high importance to recognize different types of errors. In aviation, errors can be divided into the two following categories:
- Category 1-slips and lapses which are failures in the execution of the intended action. Slips are actions that do not go as planned, while lapses are memory failures. For example, operating the flap lever instead of the (intended) gear lever is a slip. Forgetting a checklist item is a lapse;

- Category 2-mistakes which are failures in the plan of action. Even if execution of the plan was correct, it would not be possible to achieve the intended outcome. Plans that lead to mistakes can be defective (not good for anything), inappropriate (good for another situation), clumsy (with side effects) or dangerous (with increased risks).

Those two categories are unintentional and should be separated from intentional errorsviolations when one deliberates violation of a rule, procedure or norm.

Previous discussions of SHELL model, HFACS and Swiss-Cheese model, show that human error will always be present in commercial aviation. Consequently, error management strategies are crucial to controlling accident rates. Over the years, three strategies have been developed based on contemporary models and human factor research. These strategies include: Crew Resource Management (CRM), Line Operations Safety Audits (LOSA), and Threat and Error Management (TEM).

(Airbus, 2004) defines operational and human factor markers which are grouped into following clusters:

- Situation recognition and crew diagnosis-cockpit alerts, other cockpit / cabin effects, crew diagnosis, humanmachine-interface aspects; 
- Procedures-type of procedure, access to procedure, procedure contents;

- Human performance-procedure execution by flight crew, other crew actions, threat management, crew-error management, aircraft attitude / flight path control, crew coordination;

- Operating environment and circumstances-operation a 1 environment, weather conditions, runway conditions, aircraft systems condition / configuration, crew factors, organizational factors.

\section{Safety Culture}

A safety culture is the natural consequence of having humans in the aviation system. Safety culture has been described as "how people behave in relation to safety and risk when no one is watching" (ICAO, 2018). Generally, it is an expression of how safety is perceived, valued and prioritized by management and employees in an organization and it is affected by the mixture of other cultures since no human endeavor is culture-free (ICAO, 2009):

- National culture encompasses the value system of particular nations;

- Organizational / corporate culture differentiates the values and behaviors of particular organizations (e.g. government vs. private organizations);

- Professional culture differentiates the values and behaviors of particular professional groups (e.g. pilots, ATC controllers, maintenance engineers, aerodrome staff, etc.).

No human is exempt from the influence of these cultures and the safety culture can therefore be neutral, positive and negative. Its essence is what individuals believe about the importance of safety in their working environment since it is generated "topdown". Safety culture cannot be "mandated" or "designed", it evolves.

Thus, organizations should also set up a SMS and to construct safety culture in order to achieve acceptable system performance in terms of safety. The problem could occur in the practice when some aviation organizations assume that their services are already safe enough since the aviation is the safest transport mode according to global statistics. Therefore, (ICAO, 2013) provides standards and recommended practices to introduce SMS in the aviation organization by Annex 19.

\subsection{Positive Safety Culture}

An effective way to promote safe aircraft operations depends on the extent to which the operator has developed a positive safety culture. Therefore, the entire staff must be responsible for safety in relation to each being undertaken action or executed operation. Such a way of thinking must be deeply entrenched so that it really becomes a "culture".

A positive safety culture includes the following features (ICAO, 2018):

- Managers and employees, individually and collectively, want to make decisions and take actions that promote safety;

- Individuals and groups continually critique their behaviors and processes and welcome the critique of others searching for opportunities to change and improve as their environment changes;

- Management and staff share a common awareness of the hazards and risks faced 
by the organization and its activities and the need to manage risks;

- Individuals act and make decisions according to a common belief that safety is part of the way they do business;

- Individuals value being informed, and informing others, about safety;

- Individuals trust their colleagues and managers with information about their experiences and the reporting of errors and mistakes is encouraged to improve how things are done in the future.

Table 2 summarizes the organization's three responses to safety occurrences ranging from negative (pathological), through neutral (bureaucratic) to an ideal positive (generative) organizations and management of information based on safety culture principles (ICAO, 2005; ICAO, 2009).

Table 2

Three Possible Aviation Organizations and Safety Cultures

\begin{tabular}{|l|c|c|c|}
\hline Aviation Organization & Pathological & Bureaucratic & Generative \\
\hline Hazard Information & Hidden & Ignored & Sought \\
\hline Safety Messengers & Shouted & Tolerated & Trained \\
\hline Responsibility for Safety & Shirked & Boxed & Shared \\
\hline Safety Reports & Discouraged & Allowed & Rewarded \\
\hline Failures & Covered up & Merciful & Scrutinized \\
\hline New Ideas & Crushed & Problematic & Welcomed \\
\hline Resulting Organization & $\begin{array}{c}\text { Conflicted } \\
\text { organization }\end{array}$ & $\begin{array}{c}\text { "Red tape" } \\
\text { organization }\end{array}$ & Reliable \\
organization
\end{tabular}

Based on the elements shown, a positive safety culture can be described as (Čokorilo, 2017):

- Just culture-people are encouraged (even rewarded) for providing essential safety-related information. However, there is a clear line that differentiates between acceptable and unacceptable behavior;

- Reporting culture-people are prepared to report their errors and experiences;

- Informed culture-people are knowledgeable about the human, technical, organizational and environmental factors that determine the safety of the system as a whole;

- Flexible culture-people can adapt organizational processes when facing high temporary operations or certain kinds of danger, shifting from the conventional hierarchical mode to a flatter mode;

- Learning culture-people have the willingness and the competence to draw conclusions from safety information systems and the will to implement major reforms.

With the recent prominence of SMS has come a focus on safety culture, but this is 
in fact just one way in which culture can affect safety.

\section{Pilot Education, Coaching and Beyond: Building Capacity in the Cockpit}

The education of professional pilots is primarily focused on the development of their skills and piloting techniques. The introduction of SMS into the concept of aviation organizations has led to the reduction of human errors. Creating safety culture has also greatly contributed to strengthening the general awareness of the safety risks of all participants in the implementation of aviation operations and hence the pilots. What are the further possibilities for improving the work conditions for pilots and reducing errors? How to increase pilot resilience in performing daily activities and operations?

Pilot training is largely based on mentoring and practical training skills regarding to piloting certain type of aircraft, emergency situations, working in a specific work environment, etc. Today, there are a lot of opportunities for selecting powerful tools that will enable further improvement of the work conditions of each individual pilot and strengthening his personal capacities. This raises the threshold of resistance in stressful situations that can occur on every flight from technical, weather or any other reasons and impacts (nature, technology, human factor, etc.). Nowadays, the set of standards and rules due to the resting time, drinking alcohol, etc. are established by international and national regulations. On the other hand, these rules are the platform for using new tools and models in order to strengthen the pilot's personal capacities.

Each pilot has his own developmental performances and limitations. The difference between personal capabilities and performances is the field of performing modern coaching techniques. Although at the first glance such an approach may seem to outstrip business frameworks, the impact of private and business environments could have significant repercussions on pilots' performance in daily activities. Therefore, it is not enough to deal only with limitations, but to strengthen existing personal capacities, primarily through performing adequate couching business models. Today there are numerous professional coaching schools and academies in the world that perform the same or similar principles, predominantly based on a particular coaching model.

According to author's professional experience, as mentor and coach, in such complex professions, it is crucial to chose appropriate coaching model based on the client (pilot), rather than applying the same model to all clients. Therefore, key models that can easily be used in couching professional pilots are GROW and CoActive. Figure 2 shows the models and their basic characteristics. 


\begin{tabular}{|c|c|}
\hline & $\begin{array}{l}\text { What do you want? } \\
\text { What will that get you? } \\
\text { What is ex citing about this goal? } \\
\text { What's even m ore im portant than this goal? } \\
\text { How will you m ea sure the results? } \\
\text { What does success look like? } \\
\text { What'sthe big picture? }\end{array}$ \\
\hline & $\begin{array}{l}\text { How are things going right now? } \\
\text { How do you feet? } \\
\text { What values and needs are the most im portant? } \\
\text { What is the biggest concern? } \\
\text { What resources are available? } \\
\text { What barriers do you face? } \\
\text { What doesthe resistance really mean? }\end{array}$ \\
\hline pti & $\begin{array}{l}\text { What are some of the ways you could approach thisissue? } \\
\text { Would you like to brainstorm some options? } \\
\text { If you had more money, time or authority, what would you do? } \\
\text { What if you could start all over? } \\
\text { What are some of the advantages and disadvantages of each option? }\end{array}$ \\
\hline $\mathbf{W}_{\text {ay }}$ & $\begin{array}{l}\text { Which option is your best choice? } \\
\text { When will you get started? What's the first step? } \\
\text { What else do youneed to do? } \\
\text { On a scale of } 1-10 \text {, how com m itted are you to this plan? } \\
\text { What would take you to ten? } \\
\text { What accounta bility structures would support you? } \\
\text { How will you celebrate success? }\end{array}$ \\
\hline
\end{tabular}

Fig. 2

GROW vs. Co-Active Coaching Model

The GROW model is more target oriented and it is likely to find wider usage among pilots, with regard to their professional orientation, training, reasoning and decision-making principles. Therefore, it is possible to consider a number of options and to choose the best solutions and to increase the level of resilience which is of high importance during the flights, especially in emergency situations.

Therefore, it is expected that this reasoning algorithm would be transferred to other aspects of life, so the GROW model will probably be one of the most commonly used models in practice when it comes to life coaching or business coaching of professional pilots.

If a pilot strives for abstract thinking, confronting certain life and professional challenges will probably be directed to the

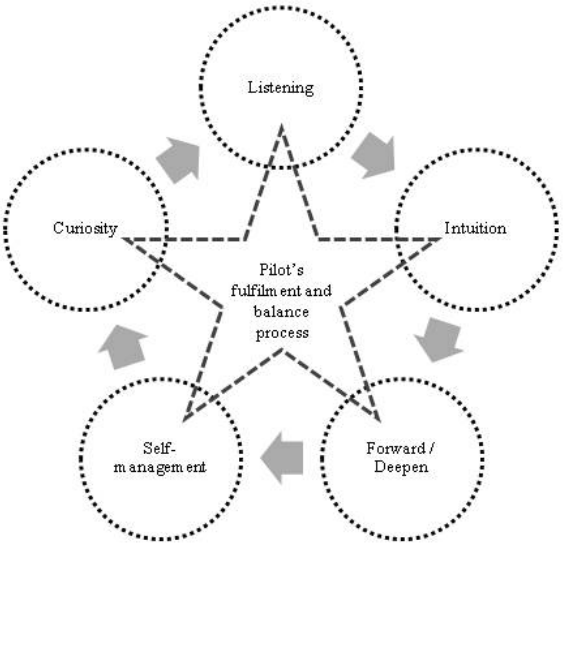

Co-Active model. This model allows the client to be placed at the center and balancing the process from this position. Regardless of the pilot's performance and capabilities, both models can deliver outstanding results in addition to mentoring process while the coach tries to empower and raise pilot resilience.

Resilience is the ability to overcome life's challenges and changes in line with personal development and progress. It is also defined as the ability of adaptation, recovery and further growth.

Pilot profession requires a whole range of changes, from organizational to private. For this reason, it is important to build a healthy relationship due to new life changes, that is, to establish a connection between the way in which the change and the stress that this change 
causes is affected. For example, overnight stay, long retention in another country outside the family, family problems, etc.

For each individual, change is the only constant in life and on the other hand, all changes are related to loss, as each loss requires a change (Neimeyer, 2000). The effects of stress depend on: the meanings that we give to certain events, the possession of personal skills for adaptation to the continuous changes, retention of the inner balance and the sense of coherence.

(Reivich and Shatté, 2002) provide one of the most used definition of resilience:

- The ability to carry with, and the regulation of emotions;

- Optimism;

- Flexible thinking;

- Empathy;

- Openness and readiness to connect with others;

- Independence and self-confidence to realize the goals.

The development of professional aviation staff requires raising their resilience. This type of support allows pilots to have a strong backbone that would allow them to cope better with stress during normal and emergency operations and to expand their capacities for changes and resilience.

Resilience of pilots in the airline company enables to maintain the level of quality and efficiency during the period of increased pressure and to recover after facing difficult situations. Therefore, pilots that are more resilient are more satisfied, healthier and more successful in realizing aviation operations.
In addition to standard training which requires compliance with rigid procedures, standards and recommended practices, the increase in pilots resilience affects the personal ability to positively adapt to: pressure, problems, challenges and changes and therefore to achieve high results, both on private and professional level.

Performing adequate coaching models to the pilots among other benefits increases their resilience and therefore affects their personal performances. The key elements are:

- Self-confidence-what is a person's belief that he has the ability to deal with the problems and challenges he encounters privately and professionally;

- Optimism-what is the person's conviction that in the end will be a good outcome and how he explains the problems he encounters;

- Setting and achieving goals-how clear is goal and a commitment to fulfill it;

- Adaptability-the ability to adapt behavior and approach, into the changing circumstances;

- Creativity in problem solving-how creative solutions are found for the problems that are encountered;

- Relationship to challenges-how much a person enjoys the challenges and are those situations recognized as an opportunity for personal growth and development;

- Emotional regulation-how much a person is able to stay calm and control his emotions in stressful situations;

- Looking for support-how much a person is ready to ask others for help and support when dealing with difficult situations. 
Choosing an adequate coaching model and coaching pilots during their professional training (combined with mentoring) and later, during the professional careers, give long-term positive results to human performance and limitations and consequently, the development of positive safety culture within the aviation company and other professional staff. Implementation of effective coaching into the professional pilots training and education will be the future in aviation safety protection.

\section{References}

Airbus. 2004. Human Performance-Human Factors Aspects in Incidents / Accidents. Flight Operations Briefing Notes. Airbus, France. 10 p.

Čokorilo, O. 2013. Human Factor Modelling for FastTime Simulations in Aviation, Aircraft Engineering and Aerospace Technology 85(5): 389-405.

Čokorilo, O.; Dell'Acqua, G. 2013. Aviation Hazards Identification Using Safety Management System (SMS) Techniques. In Proceedings of 16 th International conference on transport science (ICTS 2013). Portoroz, Slovenia, 66-73.

Čokorilo, O. 2017. Aircraft Safety. University of Belgrade, Faculty of Transport and Traffic Engineering, Belgrade, Serbia. 158 p.

ICAO. 2005. ICAO Accident Prevention Programme. International Civil Aviation Organization, Montreal, Canada. 340 p.

ICAO. 2009. Safety Management Manual-Doc 9859. $2^{\text {nd }}$ edt. International Civil Aviation Organization, Montreal, Canada. 264 p.

ICAO. 2013. Safety Management - Annex 19. $1^{\text {st }}$ edt. International Civil Aviation Organization, Montreal, Canada. 44 p.
ICAO. 2018. Safety Management Manual-Doc 9859. $4^{\text {th }}$ edt. International Civil Aviation Organization, Montreal, Canada. 170 p.

FAA. 2009. Risk Management Handbook. Federal Aviation Administration, Washington, USA.

Heinrich, H.W. 1941. Industrial Accident Prevention. A Scientific Approach. 2nd edt. New York \& London: McGraw-Hill Book Company, Inc. 448 p.

Miller, G.A. 1956. The magical number seven, plus or minus two: Some limits on our capacity for processing information, Psychological review 63(2): 81-97.

Neimeyer, R.A. 2000. Narrative disruptions in the construction of the self. In R. A. Neimeyer \& J. D. Raskin (Eds.), Constructions of disorder: Meaning-makingframeworks for psychotherapy (pp. 207-242). Washington, DC, US: American Psychological Association.

Reivich, K.; Shatté, A. 2002. The resiliencefactor: 7 essential skills for overcoming life's inevitable obstacles. Broadway Books.

Reason, J. 2016. Managing the risks of organizational accidents. Routledge. 272 p.

Reason, J. 1990. Human error. Cambridge University Press.

Rodrigues, C.; Cusick, S. 2012. Commercial Aviation Safety. Sth edt. The McGraw-Hill Companies, USA.

Shappell, S.A.; Wiegmann, D.A. 2009. A methodology for assessing safety programs targeting human error in aviation, The International Journal of Aviation Psychology 19(3): 252-269.

Wiegmann, D.A.: Shappell, S.A. 2017. A human error approach to aviation accident analysis: The human factors analysis and classification system. Routledge.

\section{jitte 14}

